doi:10.17659/01.2017.0059

Journal of Case Reports 2017;7(2):214-218

\title{
Spontaneous Regression of a Suspected Temporal lobe Glioblastoma Multiforme and its Re-appearance at a Different Site
}

Charandeep Singh Gandhoke ${ }^{1}$, Mohammad T Ansari ${ }^{1}$, Simran K Syal ${ }^{2}$, Daljit Singh ${ }^{1}$, Ravindra Kumar Saran ${ }^{3}$

Departments of ${ }^{1}$ Neurosurgery and ${ }^{3}$ Pathology, Maulana Azad Medical College and G.B. Pant Institute of Post Graduate Medical Education and Research (GIPMER), New Delhi; ${ }^{2}$ Department of Pediatrics, Sri Guru Ram Das Institute of Medical Sciences and Research, Amritsar, India.

\section{Corresponding Author:}

Dr. Charandeep Singh Gandhoke

Email: charandeepsingh2008@gmail.com

This is an Open Access article distributed under the terms of the Creative Commons Attribution License (creativecommons.org/ licenses/by/3.0).

Received

Accepted

Published

March 20, 2017

May 22, 2017

June 15, 2017

\begin{abstract}
Background: Spontaneous regression of cancer is rarely seen in brain tumors. We report a spontaneous regression of a suspected temporal lobe glioblastoma multiforme (GBM) and its re-appearance at a different site within a period of six months. Case Report: A 45 year old male presented with complaint of headache since four months and nausea, vomiting and blurring of vision since last one month. Radiological imaging was suggestive of a right temporal lobe lesion most probably high grade glioma. The patient did not undergo surgery due to personal reasons. He presented again, six months later, with exaggeration of previous symptoms. Fresh radiological imaging revealed a right parietal lobe lesion with no lesion in the previous site. Patient underwent surgery and histopathological examination was suggestive of grade IV glioblastoma. Conclusion: Spontaneous regression of a glioblastoma, to our knowledge, has never been reported in the world literature. This case report highlights the need to look for newer aspects of tumor biology in GBM.
\end{abstract}

Keywords: Brain Neoplasms, Glioblastoma, Headache, Parietal Lobe, Temporal Lobe.

\section{Introduction}

Spontaneous regression of cancer is a rare phenomenon. It is even rarer in patients suffering from brain tumors. Spontaneous regression of a glioblastoma, to our knowledge, has never been reported in the world literature. We report a spontaneous regression of a suspected temporal lobe glioblastoma multiforme and its re-appearance at a different site within a period of six months.

\section{Case Report}

A 45 year old male presented in August 2015 with chief complaints of intermittent holocranial headache since four months. This was accompanied by nausea and vomiting on and off since last one month. Patient also complained of blurring of vision since last one month. Patient was a known case of diabetes mellitus and was on regular medications.
On examination, he was conscious, oriented and had no motor deficit. Fundus examination showed bilateral papilledema. His blood profile for biochemistry and hematology was within normal limits. Tests for Human Immunodeficiency Virus (HIV), hepatitis B surface antigen and hepatitis C were negative. Non contrast computed tomography (CT) scan of the brain was suggestive of a hypodense mass lesion in the right temporal lobe with peri-lesional edema and mass effect [Fig.1]. Contrast enhanced magnetic resonance imaging (CEMRI) of the brain was suggestive of a mass lesion of size $30 \times 30 \times 35 \mathrm{~mm}$ in the right temporal lobe with enhancement of peripheral rim [Fig.2]. On magnetic resonance spectroscopy (MRS), voxels from the periphery of the lesion showed choline peak with elevated choline/creatinine ratio. The provisional diagnosis following radiological imaging was 'high grade right temporal lobe 
glioma'. The patient was advised to undergo craniotomy and tumor decompression. Due to personal reasons, the patient opted out of surgery. He was lost to follow up for 6 months. He did not take any kind of treatment during this period.

He presented again, in January 2016, with the same complaints as before but the severity of the complaints had increased. To our surprise, a fresh radiological imaging revealed a right parietal lobe lesion with no lesion in the previous site i.e. right temporal lobe [Fig.3]. Fresh contrast enhanced CT scan and CEMRI of the brain were suggestive of an ill marginated, heterogeneous mass lesion of size $50 \times 50 \times 45 \mathrm{~mm}$ with irregular peripheral enhancement and central necrosis in the right parietal lobe with peri-lesional edema, mass effect and midline shift of $10 \mathrm{~mm}$ towards the left side [Fig.3]. MRS showed a choline peak with elevated choline/creatine ratio. These features were suggestive of "high grade right parietal lobe glioma". Right parietal craniotomy and tumor decompression was done.

On histopathological examination, sections showed a cellular tumor composed of astrocytes arranged in sheets. Tumor cells were pleomorphic with high nuclear:cytoplasmic (N:C) ratio, hyperchromatic nuclei with prominent nucleolus. Endothelial proliferation and large areas of necrosis were noted. Frequent mitotic figures (6-7/10 high power fields) were seen. These findings were suggestive of glioblastoma (WHO Grade IV) [Fig.4]. The patient also received adjuvant concurrent chemo-radiotherapy, followed by 6 cycles of oral temozolomide chemotherapy.

\section{Discussion}

Glioblastoma multiforme (GBM) is the most common primary brain tumor and it is also the most malignant astrocytoma. Glioblastoma accounts for $52 \%$ of all primary brain tumors [1]. The term 'glioblastoma multiforme' was coined in 1926 by Percival Bailey and Harvey Cushing, based on
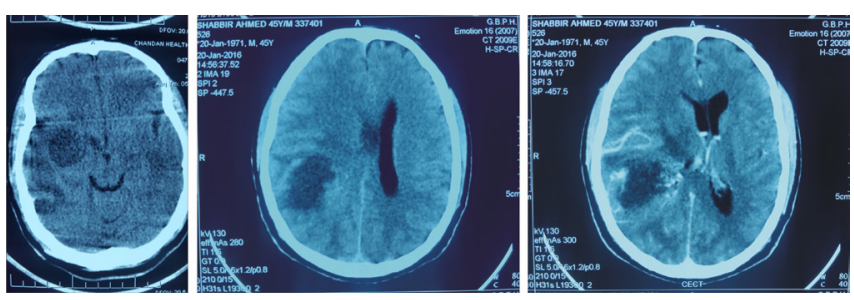

Fig.1: Non contrast Computed Tomography (NCCT) scan of the brain in August 2015 showing a hypo-dense mass lesion in the right temporal lobe. Contrast enhanced CT scan of the brain in January 2016 in the same patient showing no lesion in the right temporal lobe with the appearance of a new lesion in the right parietal lobe.

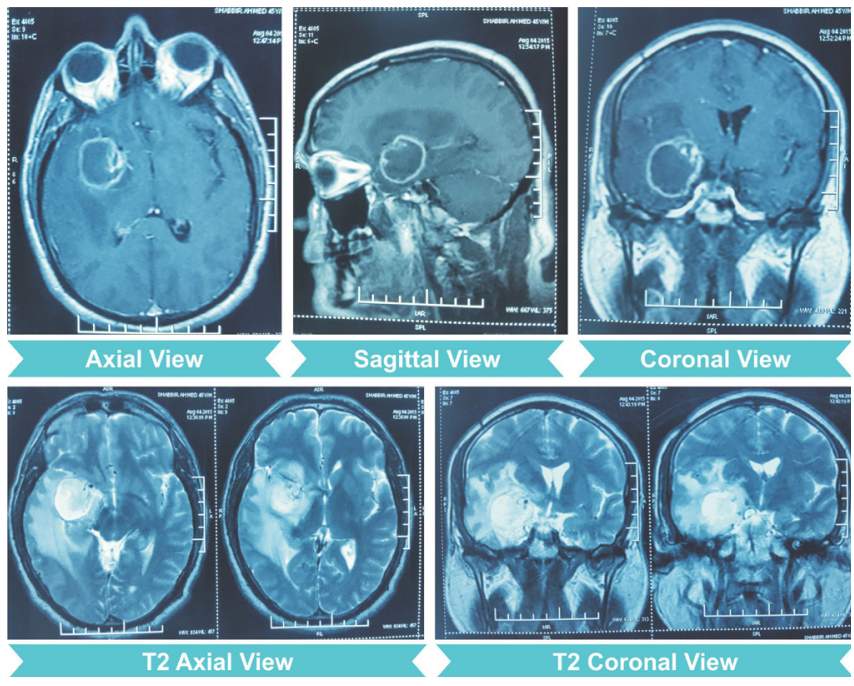

Fig.2: Contrast enhanced Magnetic Resonance Imaging (CEMRI) of the brain in August 2015 showing a ring enhancing lesion with irregular margins and peri-lesional edema in the right temporal lobe.

the idea that the tumor originates from primitive precursors of glial cells (glioblasts) and the suffix 'multiforme' was used to describe the variable appearance of these tumors due to the presence of necrosis, hemorrhage and cysts [2].

GBM can arise 'de novo' (primary GBM) or evolve from lower grade astrocytomas (secondary GBM). Primary glioblastoma is more aggressive and tends to occur in older patients. These tumors are characterized by Epidermal Growth Factor Receptor (EGFR) amplification and/or overexpression, Phosphatase and TENsin 

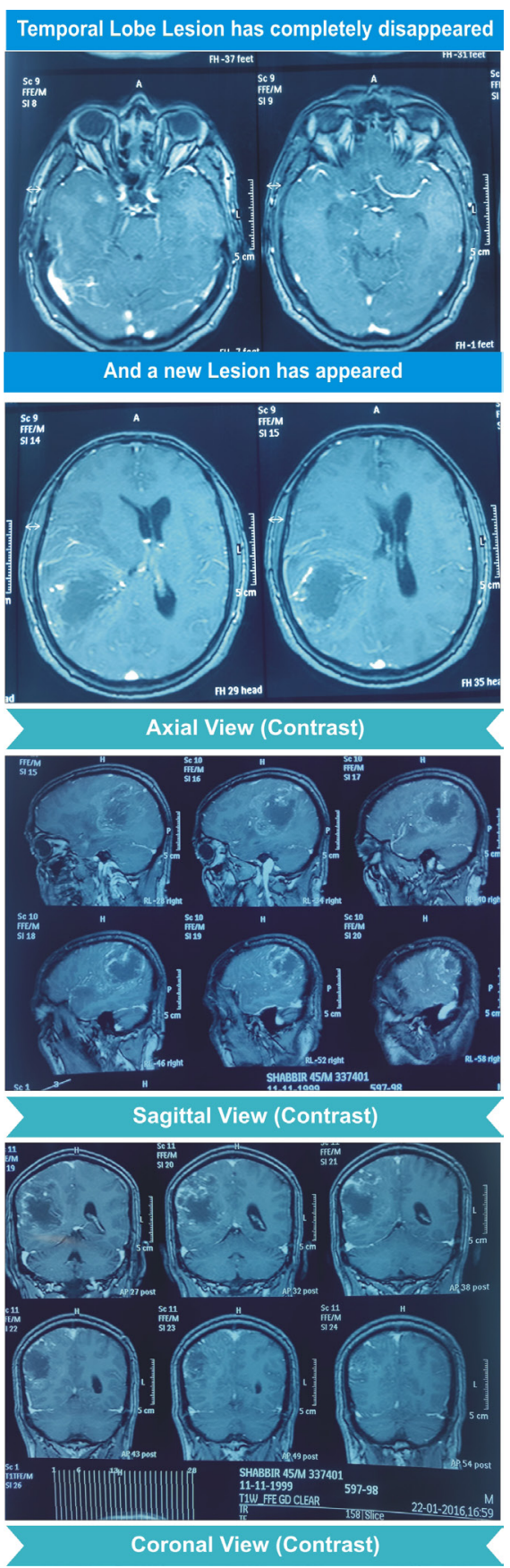

Fig.3: Contrast enhanced Magnetic Resonance Imaging (CEMRI) of the brain in January 2016 in the same patient showing no lesion in the right temporal lobe with appearance of a new lesion in the right parietal lobe.

homolog (PTEN) mutations, Mouse Double Minute 2 homolog (MDM2) overexpression and/ or loss of heterozygosity of chromosome $10 \mathrm{p}$ [3]. Secondary glioblastoma is less aggressive and occurs in younger patients. These tumors

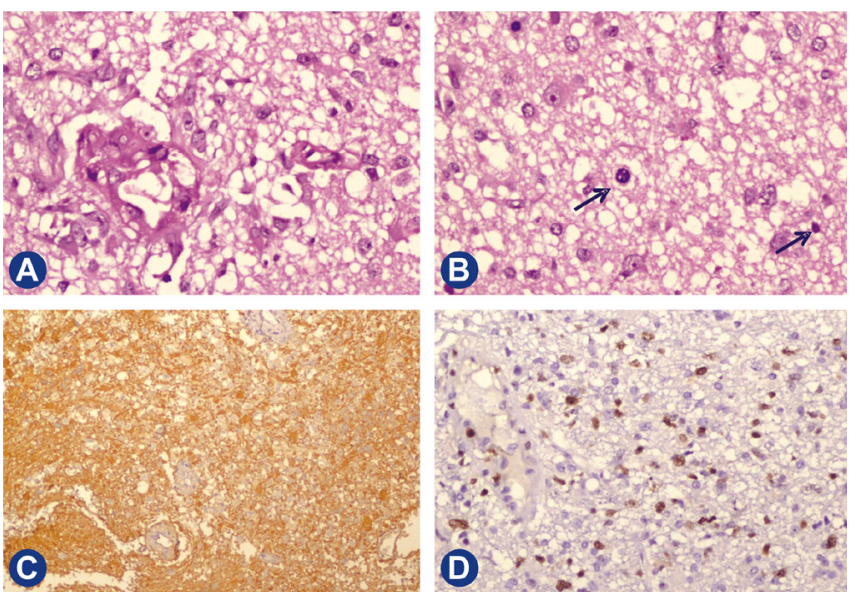

Fig.4(A): Hematoxylin \& Eosin staining showing an astrocytic tumour with vascular endothelial cell proliferation (200X); (B): showing frequent mitosis (arrow) (200X); (C): Astrocytic cells are positive on GFAP immunostaining (100X); (D): MIB labelling index is more than 15\% (100X).

are characterized by Isocitrate dehydrogenase 1 (IDH 1) mutations, p53 mutations, amplification of Platelet Derived Growth Factor A (PDGF-A) and allelic loss of chromosomes $19 q$ and $10 q$ [3]. Over $80 \%$ of secondary glioblastomas carry a mutation in IDH 1, whereas this mutation is rare in primary glioblastomas (5-10\%) which helps in differentiating between primary and secondary glioblastomas $[3,4]$. In our case, the lesion appeared 'de novo' hence it is a case of primary GBM.

Glioblastomas are multifocal in $20 \%$ of the cases and may be rarely multi-centric. Necrosis and micro-vascular proliferation are hallmarks of World Health Organization (WHO) grade IV glioblastomas [5]. Surgery followed by concurrent chemo-radiation is the treatment of choice for GBM. This is followed by at least 6 cycles of oral temozolomide chemotherapy. Prognosis is dismal. Despite treatment, the median survival time following diagnosis is 12 to 15 months [6]. Patients with both IDH 1 mutation and O6 methylguanine DNA methyl-transferase (MGMT) methylation have the longest survival; patients with either one of the above have intermediate survival and patients with neither of the above have the worst prognosis 
[7]. As we do not have a histopathological diagnosis of the disappeared temporal lobe lesion, we will consider the differential diagnosis of a ring enhancing lesion on MRI in the temporal lobe. The differential diagnosis includes cerebral abscess, CNS tuberculoma, radiation necrosis, metastasis, tumefactive demyelinating lesion, CNS lymphoma and glioblastoma.

Our patient did not have any history suggestive of unsafe chronic suppurative otitis media (CSOM) or any heart disease. In brain abscess lesions, on MRI, there is restricted diffusion and on MRS, atypical peaks (acetate, aspartate and succinate) are seen and choline peak is depressed [8]. In the above mentioned case report, on MRI, the lesion showed free diffusion of water and on MRS, the periphery of the lesion showed a choline peak. Thus, the diagnosis of cerebral abscess is highly unlikely.

There is no past history of tuberculosis or radiation exposure and the patient has not taken antitubercular treatment. The work up for metastasis was also normal in this patient. Tumefactive demyelinating lesion is a locally aggressive form of demyelination usually manifesting as a solitary lesion greater than $2 \mathrm{~cm}$ that may mimic a neoplasm on imaging. On contrast enhanced MRI, it shows an open ring pattern of enhancement with the incomplete portion of the ring on the gray matter side of the lesion. On MRI, peripheral ring enhancement in patients harbouring a $\mathrm{CNS}$ lymphoma is seen only in immuno-compromised patients (HIV/AIDS). Thus the most probable diagnosis of this spontaneously regressed temporal lobe lesion radiologically is consistent with 'glioblastoma multiforme/ high grade glioma".

Spontaneous regression of cancer is defined as the partial or complete disappearance of a malignant tumor in the absence of all treatment or in the presence of therapy which is considered inadequate to exert a significant influence on neoplastic disease [9]. It is most commonly seen in cases of neuroblastoma, renal cell carcinoma, malignant melanoma and lymphoma/ leukemia [9]. Brain tumors that show spontaneous regression include low grade tumors of the hypothalamicchiasmatic region and diffuse brainstem gliomas. Lenard et al. and Thompson et al. have reported rare cases of spontaneous remission of diffuse brainstem lesions $[10,11]$. Schmandt et al. reviewed 22 patients in whom regression of astrocytomas had occurred. 20 lesions were in the hypothalamicchiasmatic region and 2 were in the brainstem [12]. Gupta et al. reported a case of medulloblastoma, which disappeared following steroid administration and a single setting of radiotherapy (8Gy) [13].

\section{Conclusion}

Spontaneous regression of a glioblastoma, to our knowledge, has never been reported in the world literature. We report a unique and miraculous spontaneous regression of a suspected temporal lobe GBM and its re-appearance at a different site within a period of six months. This case report highlights the need to look for newer aspects of tumor biology in GBM.

Contributors: CSG: manuscript writing, case management and literature search; MTA: manuscript editing, literature search, photographs and case management. SKS, DS: manuscript editing and intellectual inputs. RKS: histopathology and manuscript editing. CSG will act as guarantor. All authors approved the final version of the manuscript.

Funding: None; Competing interests: None stated.

\section{References}

1. Young RM, Jamshidi A, Davis G, Sherman JH. Current trends in the surgical management and treatment of adult glioblastoma. Annals of Translational Medicine. 2015;3:121

2. Bailey P, Cushing H. A classification of the tumors of the glioma group on a histogenetic basis: with a correlated study of prognosis. Philadelphia: JB Lippincott; 1926.

3. Ohgaki H, Kleihues P. The definition of primary and secondary glioblastoma. Clin Cancer Res. 2013;19:764772.

4. Molenaar RJ, Radivoyevitch T, Maciejewski JP, Van Noorden CJ, Bleeker FE. The driver and passenger effects of isocitrate dehydrogenase 1 and 2 mutations in 
oncogenesis and survival prolongation. Biochimica et Biophysica Acta. 2014;1846:326-341.

5. Louis DN, Ohgaki H, Wiestler OD, Cavenee WK, Burger PC, Jouvet A, et al. The 2007 WHO classification of tumours of the central nervous system. Acta Neuropathol. 2007;114:97-109.

6. Gallego O. Nonsurgical treatment of recurrent glioblastoma. Curr Oncol. 2015;22:e273-e281.

7. Molenaar RJ, Verbaan D, Lamba S, Zanon C, Jeuken $\mathrm{JW}$, Boots-Sprenger $\mathrm{SH}$, et al. The combination of IDH1 mutations and MGMT methylation status predicts survival in glioblastoma better than either IDH1 or MGMT alone. Neuro Oncol. 2014;16:1263-1273.

8. Greenberg MS. Neuroradiology. In: Greenberg, Mark S (ed). Handbook of Neurosurgery. $7^{\text {th }}$ edition. Florida: New York, NY: Thieme Publishers;2010;133.
9. Cole WH, Everson TC. In: Spontaneous Regression of Cancer. Philadelphia, PA: WB Saunders; 1966.

10. Lenard HG, Engelbrecht V, Janssen G, Wechsler W, Tautz C. Complete remission of a diffuse pontine glioma. Neuropediatrics. 1998;29:328-330.

11. Thompson WD Jr, Kosnik EJ. Spontaneous regression of a diffuse brainstem lesion in the neonate. Report of two cases and review of the literature. J Neurosurg. 2005;102:65-71.

12. Schmandt SM, Packer RJ, Vezina LG, Jane J. Spontaneous regression of low-grade astrocytomas in childhood. Pediatr Neurosurg. 2000;32:132-136.

13. Gupta V, Kumar S, Tatke M, Singh A, Sinha S, Singh D. Disappearing cystic cerebellar medulloblastoma : the ghost tumour. Neurol India. 2001;49:291. 